\title{
Vasopressin modulates social recognition-related activity in the left temporoparietal junction in humans
}

\author{
CF Zink ${ }^{1}$, L Kempf ${ }^{1}$, S Hakimi', CA Rainey ${ }^{1}$, JL Stein ${ }^{1}$ and A Meyer-Lindenberg ${ }^{1,2}$
}

The neuropeptide vasopressin is a key molecular mediator of social behavior in animals and humans, implicated in anxiety and autism. Social recognition, the ability to assess the familiarity of others, is essential for appropriate social interactions and enhanced by vasopressin; however, the neural mechanisms mediating this effect in humans are unknown. Using functional magnetic resonance imaging (fMRI) and an implicit social recognition matching task, we employed a double-blinded procedure in which 20 healthy male volunteers self-administered $40 \mathrm{UI}$ of vasopressin or placebo intranasally, $\mathbf{4 5}$ min before performing the matching task in the scanner. In a random-effects fMRI analysis, we show that vasopressin induces a regionally specific alteration in a key node of the theory of mind network, the left temporoparietal junction, identifying a neurobiological mechanism for prosocial neuropeptide effects in humans that suggests novel treatment strategies.

Translational Psychiatry (2011) 1, e3; doi:10.1038/tp.2011.2; published online 4 April 2011

\section{Introduction}

Social recognition is a cornerstone of social cognition; the ability to properly and quickly assess the familiarity of others is essential for appropriate and beneficial social interactions. Vasopressin, a neuropeptide released in the brain that has been implicated in anxiety and autism, ${ }^{1}$ is a known molecular mediator of complex social behaviors, including social recognition. ${ }^{2,3}$ Specifically, centrally injected vasopressin in rats improves and prolongs social memory, ${ }^{4}$ whereas vasopressin receptor knockout ${ }^{5}$ and antagonism ${ }^{6}$ impairs social recognition, but not general object recognition. In agreement with these data in animals, intranasal administration of vasopressin in human males has been shown to enhance familiarity ratings of previously seen emotional faces. ${ }^{7}$

In rodents, the lateral septum is especially implicated in the action of vasopressin, through $\mathrm{V} 1 \mathrm{a}$ receptors, to influence social recognition. Re-expressing $\mathrm{V} 1 \mathrm{a}$ receptors specifically in the lateral septum of $\mathrm{V} 1 \mathrm{aR}$ knockout mice completely rescues social recognition, and overexpression of the vasopressin V1a receptor in the lateral septum of wild-type mice potentiates social recognition. ${ }^{8}$ The lateral septum is densely connected to the olfactory system in rodents, ${ }^{9}$ where social memory primarily relies on olfactory cues, while social recognition in primates, including humans, is driven by visual and auditory information. In agreement with this, social familiarity in humans has been primarily linked to cortical areas with access to multimodal visual/auditory information, such as posterior superior temporal cortex/temporoparietal junction (TPJ) and prefrontal cortex, ${ }^{10-12}$ suggesting that these regions may contribute to the (currently unknown) neural circuitry underlying the influence of vasopressin on social recognition in humans.

To test this hypothesis, in this study, using functional magnetic resonance imaging (fMRI) and an implicit social recognition matching task, we assessed the effect of vasopressin on social familiarity-related neural activity in men to determine the brain region(s) underlying the influence of vasopressin on social recognition in humans.

\section{Materials and methods}

Participants. A total of 20 right-handed, Caucasian, healthy male volunteers aged $18-43$ years (mean age $=28.60$ years, s.d. $=5.88$ ) participated in the study. Volunteers were recruited from the Washington DC Metropolitan Area and the National Institutes of Health community. Participants had no structural brain abnormalities, no history of psychiatric or neurological disorders and had normal electrocardiograms and blood pressure. Each participant gave written, informed consent for a protocol approved by the National Institute of Mental Health institutional review board.

Emotion inventories. Both before and after each of the scanning sessions, the current emotional states of the participants were assessed with the state versions of the State-Trait Anger Expression Inventory ${ }^{13}$ and State-Trait Anxiety Inventory ${ }^{14}$ to determine potential effects of vasopressin on current levels of anger and anxiety, respectively. Valence, arousal and dominance was also assessed before and after the scanning sessions using the Self-Assessment Manikin. ${ }^{15}$ Potential drug effects on each rating questionnaire were statistically determined using paired $t$-tests.

Implicit social recognition matching task. The implicit social recognition matching task used was a modified version of a block-design matching task, ${ }^{16}$ consisting of blocks matching negative emotional facial expressions and blocks

${ }^{1}$ Genes, Cognition, and Psychosis Program, NIMH, NIH, DHHS, Bethesda, MD, USA and ${ }^{2}$ Central Institute of Mental Health, Mannheim, Germany Correspondence: Dr CF Zink, National Institute of Mental Health, NIH, 9000 Rockville Pike, Building 10, Room 3C101, Bethesda, MD 20814, USA. E-mail: zinkc@mail.nih.gov

Keywords: anxiety; autism; fMRI; social recognition; temporopartietal junction; vasopressin

Received 4 March 11; accepted 6 March 11 
matching negative scene orientations as a non-social control condition-as part of a neuroimaging task battery. Subjects performed two sequential runs of the task. In both runs, the face stimuli were from the NimStim Face Stimulus Set (http:// www.macbrain.org/resources.htm), and the scene stimuli were from the International Affective Picture System (http:// csea.phhp.ufl.edu/media/iapsmessage.html). The first run ( $4.17 \mathrm{~min})$ served as training in which subjects were familiarized with two faces and two scenes that were repeatedly presented in the matching task. This training run was divided into ten blocks, five blocks of matching two faces with fearful/angry facial expressions and five blocks of matching orientation of two scenes as a non-social control condition, alternatively. Each block began with a 2-s instruction screen and consisted of four matching frames (5s each); for each matching frame, participants indicated which of the two bottom images, left or right, match the center-top image. In the second run (eight blocks: four facematching blocks and four scene-matching blocks; $3.43 \mathrm{~min}$ ), subjects performed the same task as the first run and were again presented with blocks of matching the familiar faces and scenes, but intermixed with blocks of matching unfamiliar faces and scenes (Figure 1). Two of the four matching blocks of faces and scenes used repeatedly previously seen ('familiar') stimuli, whereas in the other blocks, never before seen ('unfamiliar') stimuli were used.

Subjects performed this task (both runs) in two separate sessions, at 1 week interval, and self-administered either 40 UI of vasopressin or placebo intranasally under investigator supervision (double-blind) in each session, $45 \mathrm{~min}$ before beginning the task to capture peak CSF vasopressin levels. ${ }^{17}$ The drug order (vasopressin or placebo) was counterbalanced across subjects. The Pharmaceutical Development Section of the NIH Pharmacy Department formulated the vasopressin and placebo solutions and maintained the blind. fMRI and behavioral data are only reported for the second run of the task, as the first run served solely as training to acquire stimuli familiarity. Potential drug effects on task performance were statistically determined using paired $t$-tests and repeated-measures analysis of variance.
fMRI imaging. Scanning was performed on a 3 T GE Signa scanner (GE Healthcare, Milwaukee, WI, USA). $f M R I$ data is only reported for the second run of the social recognition matching task, as the first run served solely as training to acquire stimuli familiarity. For each participant, 98 wholebrain scans were acquired to measure the $\mathrm{T}^{*}$-weighted blood oxygenation level-dependent effect with the following parameters: gradient-recall echo planar imaging; $\mathrm{TR}=2000 \mathrm{~ms} ; \mathrm{TE}=30 \mathrm{~ms}$; flip angle $=90^{\circ} ; 64 \times 64$ matrix; $\mathrm{FOV}=240 \mathrm{~mm}$; and 28 slices (3.5-mm thick) acquired with an interleaved order of slice acquisition. Five additional scans were acquired at the beginning of run to allow for steady-state magnetization (discarded from analysis). Head movement during scanning was minimized with a vacuum pillow and additional padding.

fMRI preprocessing. fMRI preprocessing was performed using Statistical Parametric Mapping (SPM5) (http:// www.fil.ion.ucl.ac.uk/spm/). Motion correction to the first functional scan was performed using a six-parameter rigidbody transformation. For each individual, the mean of the functional images was spatially normalized to the Montreal Neurological Institute template conforming to the Talairach orientation system ${ }^{18}$ by applying a 12-parameter affine transformation followed by nonlinear warping. ${ }^{19}$ The computed transformation parameters were applied to all the functional images, interpolated to a final voxel size of $3 \times 3 \times 3 \mathrm{~mm}^{3}$. Images were subsequently spatially smoothed with an 8-mm Gaussian kernel.

fMRI analysis. A random-effects, epoch-related statistical analysis was performed in a two-level procedure using SPM5. At the first level, a separate general linear model was specified for each participant for each session. Blood oxygenation level-dependent responses during each facematching block (unfamiliar and familiar) and each scenematching block (unfamiliar and familiar) were modeled separately, by convolving the block onset vectors with a synthetic hemodynamic response function. The data were high-pass filtered (128-s cutoff) to remove low-frequency

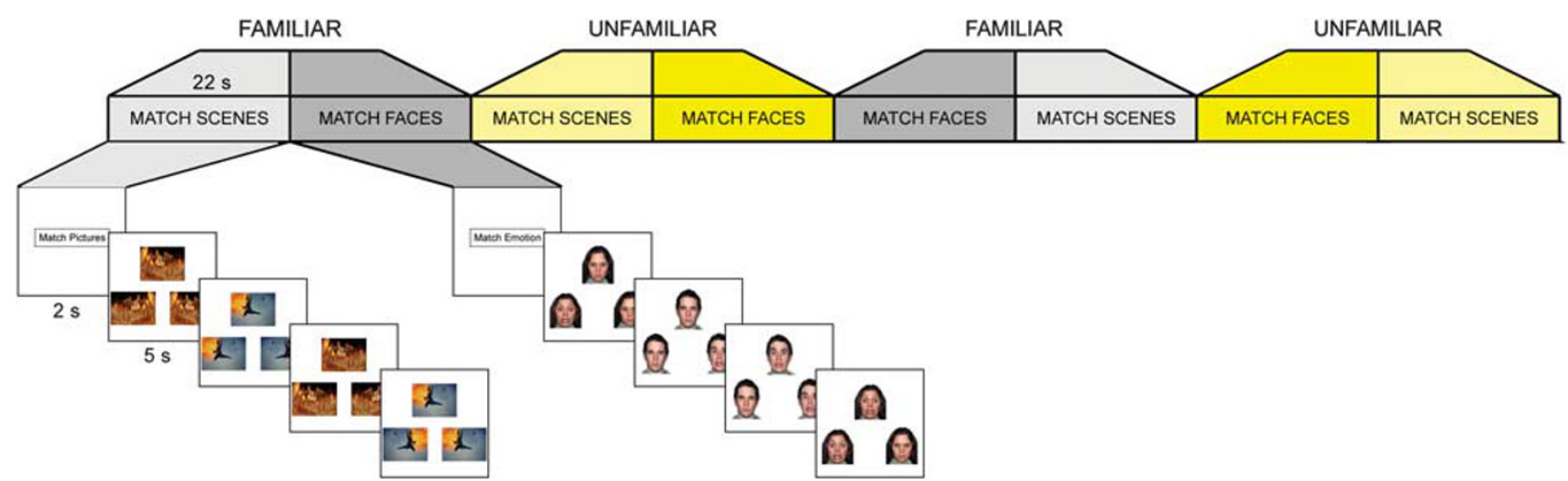

Figure 1 Schematic diagram of the social recognition matching task. The task was divided into eight blocks, four blocks of matching faces with fearful and angry facial expressions, from the NimStim Face Stimulus Set (http:/www.macbrain.org/resources.htm), and four blocks of matching scene orientation, from the International Affective Picture System (http://csea.phhp.ufl.edu/media/iapsmessage.html), as a non-social control condition. Two of the four matching blocks of faces and scenes used repeatedly previously seen ('familiar') stimuli, whereas in other blocks, never before seen ('unfamiliar') stimuli were used. Each block contained four matching panels ( $5 \mathrm{~s}$ each) and participants indicated which of the two bottom images, left or right, match with the center-top image. Instructions were displayed for $2 \mathrm{~s}$ at the beginning of each block: 'Match Emotion' or 'Match Pictures'. 
drifts, and serial correlations were accounted for by an autoregressive model of the first order. To isolate effects of social recognition, contrast images were calculated for each participant in both sessions for the interaction of sociality and familiarity ((faces-scenes) $\times$ (familiar-unfamiliar)). The individual contrast images were then entered into a second level random-effects analysis in which a paired $t$-test was used to statistically assess drug effects (vasopressin versus placebo) on resulting neural activity. A cluster-based, wholebrain family-wise error-corrected threshold of $P<0.05$ was applied to the resulting summary statistical maps using parameters determined by the Monte Carlo simulations implemented in the AlphaSim program in AFNI. ${ }^{20}$ The simulations (1000 iterations, $8 \mathrm{~mm}$ full width at half maximum, $3 \times 3 \times 3 \mathrm{~mm}^{3}$ voxels) yielded a combined threshold of $t=3$ and cluster extent of 35 contiguous voxels $\left(945 \mathrm{~mm}^{3}\right)$ as equivalent to a corrected threshold of $P<0.05$.

\section{Results}

Behavioral results. The task performance data are given in Table 1. Task performance data were not available for one subject because of technical difficulties. Participants performed the task with near-perfect accuracy, and there was no significant effect of drug on accuracy $(t=0.33$; $P=0.75$ ). Across sessions (vasopressin and placebo), there was a main effect of familiarity and sociality on reaction time periods; participants responded significantly faster when matching familiar stimuli $(\mathrm{F}(1,36)=260.71 ; P<0.001)$ and faster during face-matching blocks compared with scenes $(\mathrm{F}(1,36)=148.57 ; P<0.001)$. There was no effect of drug $(\mathrm{F}(1,36)=0.63 ; P=0.43)$.
Behavioral data from the pre- and post-scan emotional inventories are given in Table 2. The level of experienced arousal in both conditions (vasopressin and placebo) was significantly greater post-scan compared with pre-scan (placebo: $t=2.45, P=0.02$; vasopressin: $t=2.35, P=0.03$ ). No other emotional measures (valence, dominance, anger and anxiety levels) differed significantly between pre- and post-scan in either condition $(P>0.10)$. As reported previously, ${ }^{21}$ vasopressin had no significant effect on any behavioral measurements of valence, arousal, dominance, anger or anxiety ratings $(P>0.10)$.

fMRI results. Vasopressin induced a regionally specific alteration in social recognition-related activity solely in the left TPJ/Brodmann area 39 (peak: $-48,-66,21 ; t=4.81$; $k=52 ; P<0.05$ corrected; Figure $2 \mathrm{a}$ ), as demonstrated by the effect of drug compared with placebo on the interaction of sociality (faces or scenes) and familiarity (familiar or unfamiliar). Paired $t$-tests performed on beta values extracted from the peak voxel $(-48,-66,21)$ revealed that under placebo, activity in left TPJ was significantly increased by social unfamiliarity, that is, unfamiliar faces, compared with unfamiliar scenes $(t=3.42 ; P=0.003)$, an effect that vanished for familiar stimuli $(t=0.05, P=0.96)$, and under vasopressin (Figure 2b); after vasopressin, social unfamiliarity did not differentially influence TPJ activation ( $t=0.75 ; P=0.46)$ and was statistically comparable to the activation for familiar faces, compared with scenes under placebo ( $t=0.42 ; P=0.68)$. Although it does not survive statistical significance, there is also a trend for an increase in left TPJ activity to familiar social compared with non-social stimuli under vasopressin $(t=1.99 ; P=0.062)$.

Table 1 Task performance data

\begin{tabular}{lccccccccc}
\hline & \multicolumn{4}{c}{ Placebo } & & \multicolumn{2}{c}{ Vasopressin } \\
\cline { 2 - 4 } & $\begin{array}{c}\text { Unfamiliar } \\
\text { faces }\end{array}$ & $\begin{array}{c}\text { Familiar } \\
\text { faces }\end{array}$ & $\begin{array}{c}\text { Unfamiliar } \\
\text { scenes }\end{array}$ & $\begin{array}{c}\text { Familiar } \\
\text { scenes }\end{array}$ & & $\begin{array}{c}\text { Unfamiliar } \\
\text { faces }\end{array}$ & $\begin{array}{c}\text { Familiar } \\
\text { faces }\end{array}$ & $\begin{array}{c}\text { Unfamiliar } \\
\text { scenes }\end{array}$ & $\begin{array}{c}\text { Familiar } \\
\text { scenes }\end{array}$ \\
\hline $\begin{array}{l}\text { Accuracy } \\
(\% \text { correct) }\end{array}$ & $100.0(0.0)$ & $99.34(2.87)$ & $95.39(7.46)$ & $99.34(2.87)$ & $100.0(0.0)$ & $99.34(2.87)$ & $96.05(7.28)$ & $99.34(2.87)$ \\
$\begin{array}{l}\text { Reaction time } \\
(\mathrm{s})\end{array}$ & $1.43(0.31)$ & $1.25(0.30)$ & $2.10(0.41)$ & $1.55(0.39)$ & & $1.36(0.25)$ & $1.15(0.26)$ & $2.06(0.38)$ & $1.48(0.22)$ \\
\hline
\end{tabular}

Faces: data from the face-matching blocks. Scenes: data from the scene-matching blocks. Mean data are given (standard deviation in parenthesis). $N=19$; task performance data for one subject were not available.

Table 2 Behavioral data from emotion inventories

\begin{tabular}{|c|c|c|c|c|c|c|}
\hline & \multicolumn{3}{|c|}{ Placebo } & \multicolumn{3}{|c|}{ Vasopressin } \\
\hline & Pre & Post & Post-Pre & Pre & Post & Post-Pre \\
\hline $\begin{array}{l}\text { STAI-S } \\
\text { STAXI-S } \\
\text { SAM: valence } \\
\text { SAM: arousal } \\
\text { SAM: dominance }\end{array}$ & $\begin{array}{r}27.47(6.36) \\
15.30(0.66) \\
2.80(1.24) \\
6.20(1.61) \\
5.45(1.57)\end{array}$ & $\begin{array}{r}25.90(4.53) \\
15.10(0.31) \\
2.85(1.04) \\
6.95(1.36) \\
5.75(1.45)\end{array}$ & $\begin{array}{r}-1.95(5.83) \\
-0.20(0.77) \\
0.05(1.15) \\
0.75(1.37) \\
0.30(1.08)\end{array}$ & $\begin{array}{r}27.55(6.63) \\
15.45(1.23) \\
2.85(1.27) \\
5.75(1.48) \\
5.90(1.71)\end{array}$ & $\begin{array}{r}27.16(6.41) \\
15.40(0.88) \\
3.15(0.99) \\
6.65(1.81) \\
5.90(1.07)\end{array}$ & $\begin{array}{r}-0.79(5.10) \\
-0.05(0.69) \\
0.30(1.38) \\
0.90(1.71) \\
0.00(1.38)\end{array}$ \\
\hline
\end{tabular}

Abbreviations: SAM, self-assessment manikin; STAI-S, State-Trait Anxiety Inventory-State version; STAXI-S, State-Trait Anger Expression Inventory-State version. ${ }^{a}$ Arousal post-scan levels were significantly different from pre-scan levels in both the placebo $(P=0.02)$ and vasopressin conditions $(P=0.03)$.

Mean data are given (standard deviation in parenthesis). 

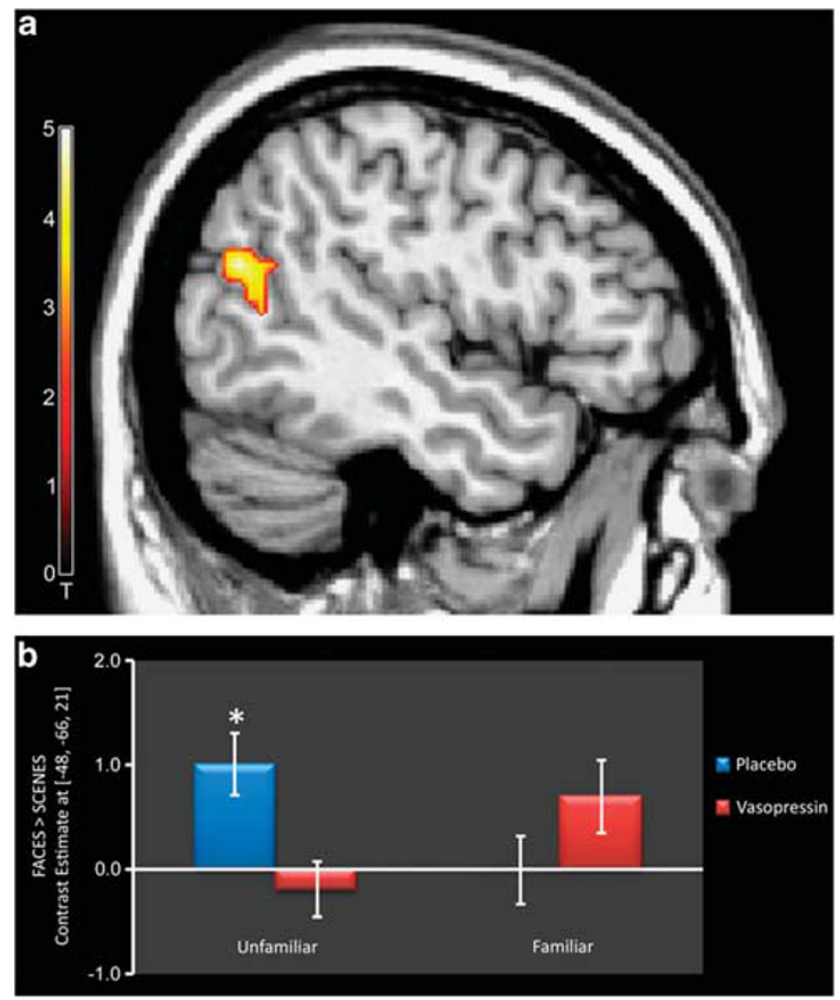

Figure 2 The effect of vasopressin compared with placebo on the interaction of sociality (faces/scenes) and familiarity (familiar/unfamiliar) revealed (a) a significant activation in the left temporoparietal junction, overlaid on a sagittal section of a structural template MRI $(x=-48)$. The color bar represents $t$-values. (b) Plotted are the contrast estimates extracted from the peak-activated voxel $(-48,-66,21)$. Bars represent means across subjects and error bars represent standard error. Under placebo (blue bars), contrast estimates were significantly greater for unfamiliar faces compared with unfamiliar scenes $\left({ }^{\star} P=0.003\right.$ ), an effect that was abolished by stimulus familiarity and vasopressin (red bars) administration.

\section{Discussion}

Our findings demonstrate that vasopressin modulates social recognition-related activity in the left TPJ in humans. During exposure to socially unfamiliar stimuli, vasopressin abolishes the augmentation of left TPJ activity evoked by social unfamiliarity under placebo to a level comparable to that evoked by socially familiar stimuli under placebo. As such, these data suggest that, when unfamiliar faces are processed (matched) under vasopressin, stimuli are more readily transferred to a familiar categorization, as represented in TPJ reactivity. The TPJ has been implicated in the processing of social background context and social familiarity ${ }^{22}$ and accesses multimodal visual/auditory information that drive social recognition in primates, including humans. ${ }^{10-12}$ Conversely, in rodents, the lateral septum, connected to the olfactory system, is a critical site of action for vasopressin to influence social recognition. ${ }^{8}$ The absence of differential activation in the lateral septum, while not excluding an effect in humans (as this structure is difficult to image in human brain), could therefore be related to species differences.

Vasopressin is likely acting on vasopressin $\mathrm{V} 1$ a receptors in the brain to influence familiarity of social stimuli; ${ }^{8}$ however, although V1a receptors are evident in various primate brain regions, ${ }^{23}$ it remains unclear if they exist in the human TPJ. Therefore, whether vasopressin is acting directly or by an indirect pathway to influence social recognition-related activity in the left TPJ remains to be elucidated.

In previous work, the directionality of the relationship between TPJ activity and social familiarity has been inconsistent (that is, activity enhanced by social familiarity or activity enhanced by social unfamiliarity). Such discrepancies may be dependent on whether familiar stimuli were newly learned, ${ }^{22}$ as here, or personally familiar ${ }^{11}$ (for example, relatives). Future work should determine whether our vasopressin finding extends to personally familiar faces. The TPJ is also a critical brain structure associated with human-specific social cognition, ${ }^{12}$ notably involved in 'theory of mind' processes, ${ }^{24}$ suggesting further studies of vasopressin effects on these aspects of social cognition as well.

Given the prominent role of the TPJ in social-specific cognition, the TPJ activation to unfamiliar faces under placebo may represent the social relevance or importance of these stimuli, relative to non-social or familiar stimuli. As such, based on the current findings, vasopressin may influence social recognition by modulating left TPJ activity to dampen the social importance of unfamiliar faces while potentially (evident by trend in TPJ activity increase) enhancing the social importance of familiar faces.

Behaviorally, vasopressin did not influence the emotion inventories or task performance. This lack of behavioral modification is consistent with previous studies in which participants performed a comparable matching paradigmbut without manipulating social familiarity-after administration of vasopressin ${ }^{21}$ and the related neuropeptide, oxyto$\operatorname{cin}^{25}$, and is likely due to the implicit nature of the task (that is, subjects were not explicitly tested on social recognition). Alternatively, it is possible that the lack of a significant influence of drug on task performance is due to a ceiling effect; therefore, implementation of a more challenging task could reveal vasopressin effects on task performance accompanying the reported drug-induced modification of neural activity.

We note that behaviorally, both oxytocin ${ }^{26}$ and vasopres$\sin ^{7}$ have been shown to increase the familiarity of faces, suggesting that this could be a joint mechanism supporting the prosocial action of these neuropeptides. On the basis of this study, it should be investigated whether familiarity after oxytocin administration is also mediated through the TPJ or rather, as previous researchers have proposed, ${ }^{26}$ through an amygdala-fusiform face area circuit.

In conclusion, to our knowledge, this is the first study to investigate the neural biology underlying the effect of vasopressin on social recognition in humans, and our data identify the first regionally specific pharmacological strategy targeting the TPJ, suggesting that vasopressin receptors could be present in human cortex or action through an as yet unspecified indirect pathway. Our results implicate a neurobiological mechanism by which vasopressin may enhance social familiarity by modulating the TPJ and open a pharmacological approach to target this region in psychiatric disorders with known social cognitive deficits such as autism and social anxiety disorder. 


\section{Conflict of interest}

The authors declare no conflict of interest.

Acknowledgements. We thank Mbemba Jabbi, Katherine $\mathrm{V}$ Roe and Tiffany A Nash for analysis assistance; Yunxia Tong and Qiang Chen for technical assistance; Timothy $\mathrm{O}$ Laumann and lan J Lent for research assistance; and Gerald Overman and Judith Starling for pharmaceutical management. This research was supported by the Intramural Research Program of the National Institute of Mental Health, $\mathrm{NIH}$.

1. Ebstein RP, Israel S, Lerer E, Uzefovsky F, Shalev I, Gritsenko I et al. Arginine vasopressin and oxytocin modulate human social behavior. Ann NY Acad Sci 2009; 1167: 87-102.

2. Caldwell HK, Lee HJ, Macbeth AH, Young WS III. Vasopressin: behavioral roles of an 'original' neuropeptide. Prog Neurobiol 2008; 84: 1-24.

3. Raggenbass M. Overview of cellular electrophysiological actions of vasopressin. Eur $J$ Pharmacol 2008; 583: 243-254.

4. Le Moal M, Dantzer R, Michaud B, Koob GF. Centrally injected arginine vasopressin (AVP) facilitates social memory in rats. Neurosci Lett 1987; 77: 353-359.

5. Bielsky IF, Hu SB, Szegda KL, Westphal H, Young LJ. Profound impairment in social recognition and reduction in anxiety-like behavior in vasopressin V1a receptor knockout mice. Neuropsychopharmacology 2004; 29: 483-493.

6. Everts HG, Koolhaas JM. Lateral septal vasopressin in rats: role in social and object recognition? Brain Res 1997; 760: 1-7.

7. Guastella AJ, Kenyon AR, Alvares GA, Carson DS, Hickie IB. Intranasal arginine vasopressin enhances the encoding of happy and angry faces in humans. Biol Psychiatry 2010; 67: 1220-1222.

8. Bielsky IF, Hu SB, Ren X, Terwilliger EF, Young LJ. The V1a vasopressin receptor is necessary and sufficient for normal social recognition: a gene replacement study. Neuron 2005; 47: 503-513.

9. Risold PY, Swanson LW. Connections of the rat lateral septal complex. Brain Res Brain Res Rev 1997; 24: 115-195.

10. Frith $U$, Frith $C D$. Development and neurophysiology of mentalizing. Philos Trans $R$ Soc Lond B Biol Sci 2003; 358: 459-473.

11. Gobbini MI, Haxby JV. Neural systems for recognition of familiar faces. Neuropsychologia 2007; 45: 32-41.

12. Van Overwalle F. Social cognition and the brain: a meta-analysis. Hum Brain Mapp 2009; 30: $829-858$

13. Spielberger CD. Manual for the State-Trait Anger Expression Inventory (STAXI). Psychological Assessment Resources: Odessa, FL, 1988.

14. Spielberger CD, Gorsuch RL, Lushene PR, Vagg PR, Jacobs AG. Manual for the StateTrait Anxiety Inventory (Form Y). Consulting Psychologists Press: Palo Alto, 1983.

15. Bradley MM, Lang PJ. Measuring emotion: The Self-Assessment Manikin and the Semantic Differential. J Behav Ther Exp Psychiatry 1994; 25: 49-59.

16. Hariri AR, Tessitore A, Mattay VS, Fera F, Weinberger DR. The amygdala response to emotional stimuli: a comparison of faces and scenes. Neuroimage 2002; 17: 317-323.

17. Born J, Lange T, Kern W, McGregor GP, Bickel U, Fehm HL. Sniffing neuropeptides: a transnasal approach to the human brain. Nat Neurosci 2002; 5: 514-516.

18. Talairach J, Tournoux P. Co-planar Stereotaxic Atlas of the Human Brain: 3-Dimensional Proportional System: an Approach to Cerebral Imaging. Thieme Medical Publishers: New York, 1988, viii, pp 122

19. Ashburner J, Friston KJ. Nonlinear spatial normalization using basis functions. Hum Brain Mapp 1999; 7: 254-266.

20. Cox RW. AFNl: software for analysis and visualization of functional magnetic resonance neuroimages. Comput Biomed Res 1996; 29: 162-173.

21. Zink CF, Stein JL, Kempf L, Hakimi S, Meyer-Lindenberg A. Vasopressin modulates medial prefrontal cortex-amygdala circuitry during emotion processing in humans. I Neurosci 2010; 30: 7017-7022.

22. Saxe R, Wexler A. Making sense of another mind: the role of the right temporo-parietal junction. Neuropsychologia 2005; 43: 1391-1399.

23. Young LJ, Toloczko D, Insel TR. Localization of vasopressin (V1a) receptor binding and mRNA in the rhesus monkey brain. J Neuroendocrinol 1999; 11: 291-297.

24. Bahnemann M, Dziobek I, Prehn K, Wolf I, Heekeren HR. Sociotopy in the temporoparietal cortex: common versus distinct processes. Soc Cogn Affect Neurosci 2010; 5: 48-58.

25. Kirsch $P$, Esslinger $C$, Chen Q, Mier D, Lis S, Siddhanti S et al. Oxytocin modulates neural circuitry for social cognition and fear in humans. J Neurosci 2005; 25: 11489-11493.

26. Rimmele U, Hediger K, Heinrichs M, Klaver P. Oxytocin makes a face in memory familiar. J Neurosci 2009; 29: 38-42.

(c)

SOMERIAHIS RE No Derivative Works 3.0 Unported License. To view a copy of this license, visit http://creativecommons.org/licenses/by-nc-nd/3.0/ 